\title{
Estudio in vitro comparativo del diagnóstico radiológico de lesiones de caries dental proximal utilizando las técnicas convencional y digital directa
}

Comparative study of the radiologic diagnosis of proximal caries lesions using conventional and direct digital techniques

Francis Marcial López Puerta ${ }^{1, a}$, Roberto Antonio León-Manco ${ }^{1, a ; 2, b}$, Jorge Arturo Beltrán-Silva ${ }^{3, c, d .}$.

\section{RESUMEN}

Objetivos: Comparar el diagnóstico radiológico de lesiones de caries proximal utilizando la técnica de aleta de mordida convencional y técnica digital directa con dispositivo de carga acoplada empleando una base de datos con los registros diagnósticos. Materiales y métodos: Se evaluó la base de datos de los diagnósticos radiológicos de 108 piezas dentarias (50 molares y 58 premolares) con 106 superficies mesiales cariadas y 100 superficies distales cariadas. Esta base de datos registraba el diagnóstico de caries proximal en R0, R1, R2, R3 y R4; clasificación hecha por Pitts modificada por la Universidad Peruana Cayetano Heredia. Se calculó la concordancia en el diagnóstico, obteniendo un coeficiente Kappa de $\mathrm{p}<0.01$. El estudio tuvo una significancia de $95 \%$ y un p $<0.05$. Resultados: Según la técnica de aleta de mordida convencional se encontró un 53,9\% de R0, 22,3\% de $\mathrm{R} 1,7,8 \%$ de $\mathrm{R} 2,11,2 \%$ de $\mathrm{R} 3$, y $4,9 \%$ de $\mathrm{R} 4$ y con la técnica digital directa con dispositivo de carga acoplada un 52,9\% de R0, 14,6\% de R1, 11,7\% de R2, 14,6\% de R3, y 6,3\% de R4. Conclusiones: Existe diferencia en el diagnóstico radiológico de lesiones de caries dental interproximal utilizando ambas técnicas. Sin embargo, no se puede afirmar la superioridad de alguna de ellas por falta de gold estándar histológico.

PALABRAS CLAVE: Caries dental, radiografía, radiografía dental digital, base de datos. (Fuente: DeCS)

\section{SUMMARY}

Objectives: Compare the radiologic diagnosis of proximal caries lesions using conventional bitewing technique and direct digital charge-coupled device using a database with diagnostic records. Materials and Methods: There were evaluated the radiographic diagnostic database of 108 teeth (50 molars and 58 premolars) with 106

\footnotetext{
${ }^{1}$ Facultad de Estomatología, Universidad Peruana Cayetano Heredia. Lima, Perú

2 ONG Yanapaythani. Lima, Perú.

${ }^{3}$ Asociación Peruana de Radiología Bucal y Maxilofacial. Lima, Perú

a Cirujano Dentista.

b Director Ejecutivo.

c Especialista en Radiología Oral y Maxilofacial.

a Magister en Estomatología.
} 
mesial and 100 distal carious surfaces. This database consists of proximal caries diagnosis in R0, R1, R2, R3 and R4; Pitts made by classification modified by Universidad Peruana Cayetano Heredia. The agreement was got in the diagnosis by testing Kappa coefficient was $p<0.01$. The study had a significance of $95 \%$ and $p<0.05$. Results: According to the conventional bitewing technique was found a $53.9 \%$ of R0, $22.3 \%$ of R1, $7.8 \%$ of R2, $11.2 \%$ of R3, and $4.9 \%$ of R4 and direct digital charge-couple device technique was found a $52.9 \%$ of R0, $14.6 \%$ of R1, $11.7 \%$ of R2, $14.6 \%$ of R3, and $6.3 \%$ of R4. Conclusions: There is a difference in the radiographic diagnosis of interproximal caries lesions using both techniques. However, it can not affirm the superiority of some of them for lack of histological gold standard.

\section{KEYWORDS: Dental caries, radiography, digital radiography, database. (Source: Mesh)}

\section{INTRODUCCIÓN}

A través de los años se ha podido determinar que la enfermedad más prevalente de la cavidad oral es la caries dental afectando casi el $96 \%$ de la población mundial (1). Al mismo tiempo, tanto científicos como profesionales de la salud han estudiado su origen y prevalencia para combatirla, sin embargo aún existen dificultades en su diagnóstico clínico y radiológico (2). La caries dental es una enfermedad de origen multifactorial donde la principal característica es la destrucción de los tejidos duros de los dientes como consecuencia de la desmineralización provocada por los ácidos que genera la placa bacteriana, por lo cual el diagnóstico temprano es muy importante (3).

Actualmente, con el avance de la tecnología, existen técnicas que permiten detectar la caries dental en una fase temprana. Uno de los principales beneficios que brindó la incorporación de la imagenología como herramienta indispensable en el diagnóstico y la práctica de la odontología fue, sin duda alguna, la detección de la caries dental, y determinación del grado de destrucción y penetración de cada lesión en particular(4).

La utilidad del examen radiológico reside en el hecho que sirve como un complemento para la inspección visual, debido a que muchas lesiones incipientes de las caras oclusales y superficies proximales no se pueden diagnosticar con facilidad (5). El sistema radiológico digital fue desarrollado a finales de los años ochenta con el fin de obtener imágenes 2D con una dosis de radiación menor que la radiografía convencional, ofreciendo otra alternativa que disminuya los problemas de distorsión de imágenes y manejo de la resolución (6).
El objetivo de la investigación fue comparar el diagnóstico radiológico de lesiones de caries proximal utilizando la técnica de aleta de mordida convencional y técnica digital directa con dispositivo de carga acoplada empleando una base de datos con los registros diagnósticos, teniendo como limitación sólo poder indicar la existencia de diferencia diagnostica entre ambas técnicas, debido a no contarse con un gold estándar histológico proporcionando resultados que contribuyen al conocimiento científico, siendo parte de un conjunto de investigaciones para mejorar la práctica clínica en el diagnóstico de caries dental.

\section{MATERIAL Y MÉTODOS}

Para el presente estudio la muestra fue la misma que la población. La muestra estuvo constituida por una base de datos de los diagnósticos radiológicos de 108 piezas dentales ( 50 molares y 58 premolares, con 106 superficies mesiales y 100 distales) siguiendo el mínimo muestral de 100 especímenes como en el caso de Mohtavipour (7), consta del diagnóstico radiológico de caries dental usando la técnica de aleta de mordida con radiografía convencional y técnica digital CCD (Charge Coupled Device). Los datos fueron proporcionados por el Departamento Académico de Medicina y Cirugía Bucomaxilofacial de la UPCHFE (Universidad Peruana Cayetano Heredia - Facultad de Estomatología). Es importante mencionar que el estudio forma parte de la línea de investigación del diagnóstico de enfermedades más frecuentes de la Sección de Imagenología Bucomaxilofacial; esta sección seleccionó los especímenes que previamente habían sido diagnosticados con lesiones de caries, elaboró las maquetas, hizo las tomas radiográficas, realizó los diagnósticos y construyó la base de datos.

Se excluyeron los registros incompletos de la base de 
datos de diagnósticos radiológicos de lesiones de caries dental usando la técnica de aleta de mordida convencional y técnica digital directa $\mathrm{CCD}$, al igual que las maquetas de estudio que presentaron especímenes con destrucción dentaria provocada por trauma o que presentaron algún tipo de tratamiento restaurador, de rehabilitación, de conductos, con anatomía coronaria alterada e imágenes radiográficas distorsionadas, poco nítidas o parciales. Se utilizaron dos fichas de registro en función de la base de datos de diagnósticos radiológicos de caries dental.

El primer paso fue solicitar el permiso para la utilización de la base de datos de diagnósticos radiológicos de caries dental al Departamento Académico de Medicina y Cirugía Bucomaxilofacial de la Facultad de Estomatología de la Universidad Peruana Cayetano Heredia, que constó de 108 registros, 50 molares y 58 premolares, con 106 superficies mesiales y 100 distales. Cada registro indicó el diagnóstico radiológico de caries dental usando la técnica de aleta de mordida convencional y técnica digital directa con dispositivo de carga acoplada.

Esta base de datos fue elaborada por la Sección de Imagenología Bucomaxilofacial, que seleccionó los especímenes, elaboró las maquetas, hizo la toma radiográfica, realizó los diagnósticos y construyó la base de datos. Los diagnósticos fueron realizados por dos examinadores (Gold Estándar en radiología oral y maxilofacial) que se sometieron a un proceso de calibración inter-examinador obteniendo un nivel de concordancia de Kappa $>0.90$. Luego de obtener la base de datos se pasó a verificar que todos los registros estén completos para su posterior análisis. Finalmente, se procedió a la obtención de las frecuencias relativas y absolutas de las variables y el análisis de concordancia mediante la Prueba de Kappa, se empleó el programa Excel y SPSS v. 20. El presente estudio contó con un nivel de confianza de $95 \%$ y un $\mathrm{p}<0,05$.

El estudio contó con la aprobación del Comité Institucional de Ética de la Universidad Peruana Cayetano Heredia con fecha 07 de Julio de 2015 (SIDISI N ${ }^{\circ}$ 64736).

\section{RESULTADOS}

Se seleccionó la base de datos de los diagnósticos radiológicos de 108 piezas dentales de los cuales fueron
50 molares y 58 premolares con 106 superficies proximales mesiales y 100 superficies proximales distales. Usando la radiografía convencional se encontró que el máximo valor fue $111(53,9 \%)$ superficies proximales sanas (R0) y el menor valor fue en lesiones cariosas proximales R4 con 10 (4,9\%). Los datos registrados por medio de la radiografía digital directa con dispositivo acoplado CCD presenta el máximo valor con 109 (52,9\%) superficies proximales sanas (R0) $\mathrm{y}$ el menor valor fue en lesiones cariosas proximales R4 con 13 (6,3\%). En la comparación del registro de diagnóstico de lesiones de caries dental según técnicas radiográficas se evidenció una concordancia moderada (Kappa $=0,51, \mathrm{p}<0,01)$ siendo necesaria para pruebas diagnósticas un mínimo de concordancia muy buena (Kappa $>0,80$ ). Según la concordancia del diagnóstico radiológico de lesiones cariosas proximales se encontró que existe mayor valor en lesiones R4 (100\%) seguidamente de lesiones R3 (82,6\%), R0 $(80,2 \%), \mathrm{R} 2(56,3 \%)$ y como menor valor en lesiones R1 (28,3\%) (Tabla 1).

Para la concordancia se tomó como referencia la radiografía digital, se reportó que existió una mayor concordancia de lesiones proximales de caries dental con diagnóstico R4 en piezas premolares con un 70\% en superficie distal y $20 \%$ en superficie mesial, quedando un $10 \%$ en piezas molares. El menor valor de concordancia obtenido con diagnóstico de R1 se encontró que las piezas premolares obtuvieron un 13\% y $6,5 \%$, en superficies mesial y distal respectivamente. Podemos encontrar que hubo mayor concordancia en los diagnósticos de lesiones de caries proximal de piezas premolares con valores en R0 $(32,4 \%)$, R1 $(19,6 \%)$, R2 (50\%), R3 (43,5\%) y R4 (90\%); y según superficie el mayor valor se encontró en mesial con R0 (42,3\%), R1 (15,2\%), R2 (37,5\%), R3 (43,5\%) y R4 (20\%) (Tabla 2).

Los resultados demostraron que existe una mayor prevalencia de lesiones de caries dental en piezas premolares y en las superficies mesiales según la base de datos registrada.

\section{DISCUSIÓN}

La detección de lesiones cariosas depende de un diagnóstico preciso para los odontólogos los cuales determinan el tipo de tratamiento adecuado. Existen varios métodos para detectar caries dental en una fase 


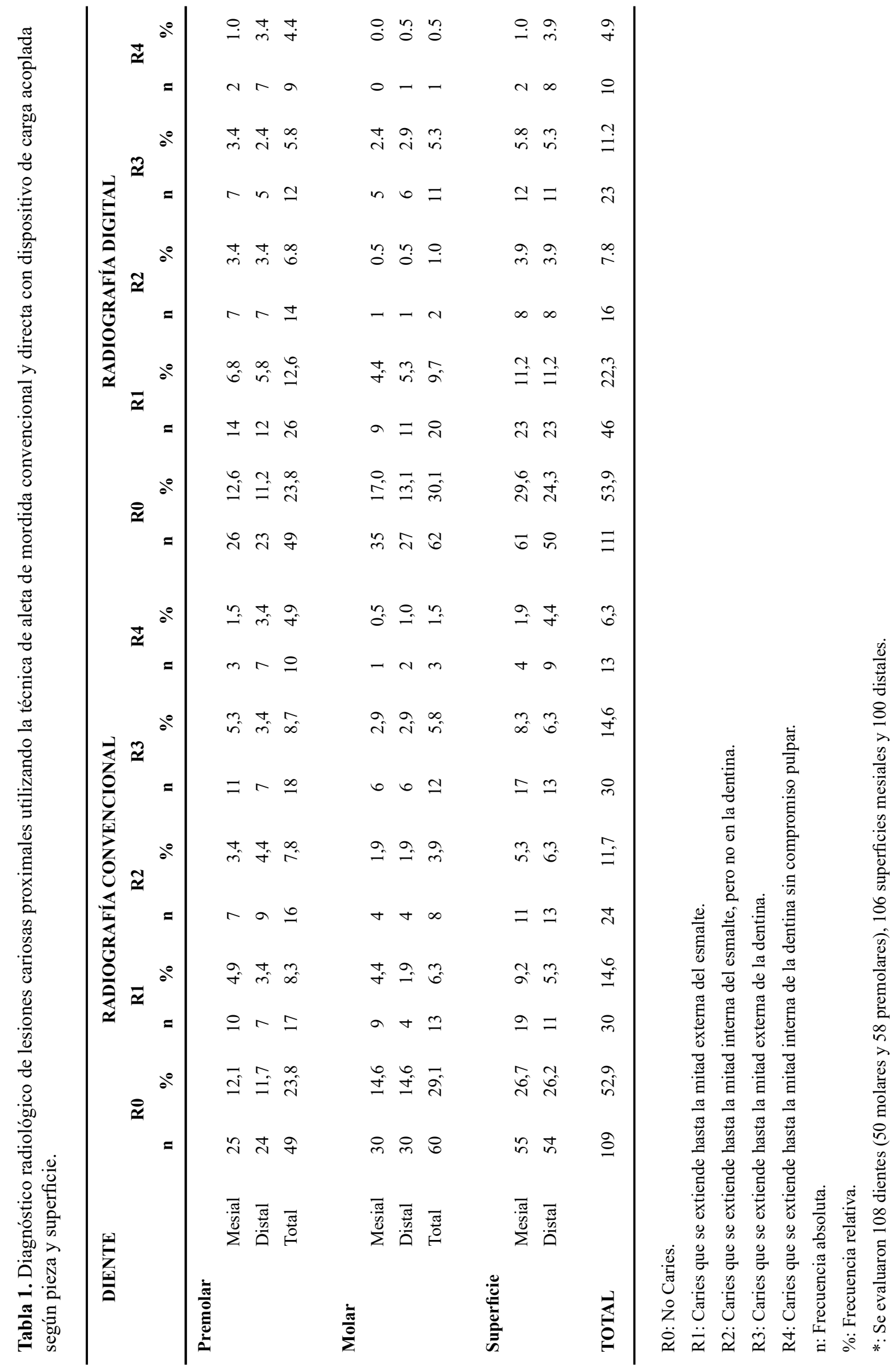




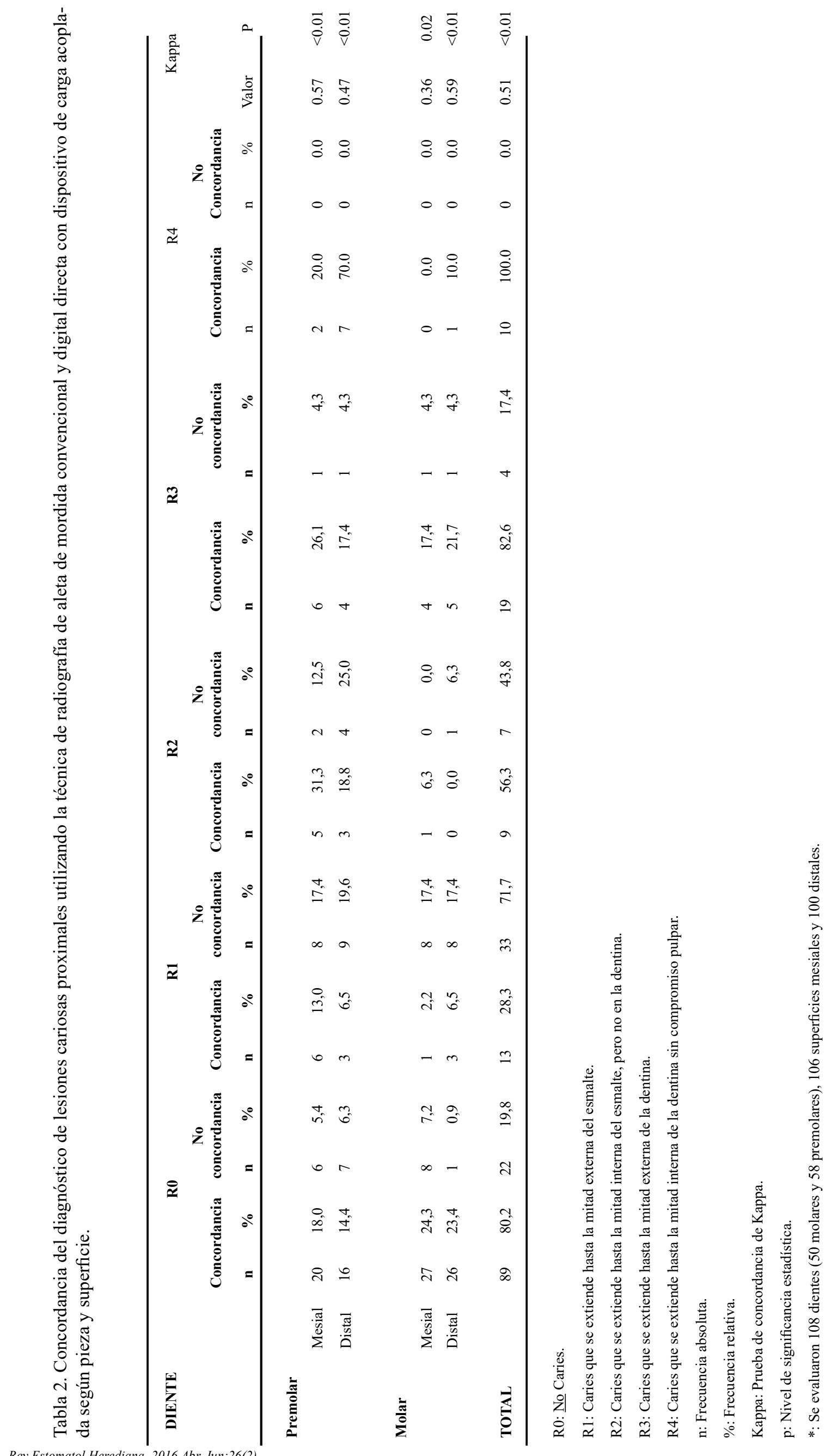


temprana antes que comprometa el tejido pulpar; la técnica radiográfica convencional es la más usada entre todos los métodos auxiliares que existen, sin embargo, cada vez se usan nuevas técnicas como la radiografía digital e incluso existen otros estudios que reportan a la tomografía computarizada como técnica diagnóstica $(8,9)$.

Por muchos años los odontólogos han usado la radiografía convencional para la evaluación de lesiones cariosas como primera opción debido a que presenta numerosas ventajas. La técnica de aleta de mordida tiene aproximadamente unos 75 años como complemento en la evaluación clínica para detectar caries proximal y se ha convertido en el examen radiológico intraoral más usado. Se puede obtener imágenes de las coronas y zonas cervicales de las piezas dentales superiores e inferiores, al mismo tiempo debido a las características de los rayos X, las imágenes obtenidas experimentan fenómenos de deformación o distorsiones verticales, horizontales, por amplitud o por desplazamiento. También permite determinar la presencia y extensión de lesiones de caries dental que no pudieron ser diagnosticadas clínicamente $(10,11)$.

La radiografía digital fue inventada por Mouyen y fue introducida al mercado en 1984 y se basa en la sustitución de la película radiográfica convencional por sensores o captadores que tienen incorporado tecnología CCD o placa de fósforo. Los datos análogos recibidos son transformados en datos digitales (sistema binario) y la computadora procesa la información en el monitor. Este sistema presenta diversas ventajas que permite facilitar el acceso a mayor información debido a una mejor resolución de contraste, al mismo tiempo se puede almacenar y enviar información a través de medios digitales, además de las herramientas que cada software provee (12).

Muhammed y Mohammed realizaron una investigación cuyo objetivo fue comparar la calidad de imagen entre dos diferentes tipo de radiografías: digitales (Sirona, Alemania y Vista-scan, Alemania) y una película E-speed (Kodak, Rochester, NY) con la técnica convencional; se seleccionaron un total de 25 dientes extraídos que fueron evaluados por dos observadores.

Los resultados revelaron que existió una diferencia significativa entre los diferentes tipos de imágenes digitales con una mayor calidad en el sistema Siro- na que el Vista-scan, sin embargo se obtuvo mayor precisión en el diagnóstico con la técnica convencional (13). La presente investigación obtuvo resultados similares, ya que encontramos diferencias estadísticamente significativas para el diagnóstico radiológico de lesiones de caries dental proximal entre la técnica radiográfica aleta de mordida realizada con radiografías convencionales y la realizada con sistema digital CCD.

En el estudio de Anupama y col., decidieron comparar la radiografía convencional y el sistema digital Dexis para evaluar la precisión en el diagnóstico de lesiones de caries proximal; esta investigación se llevó a cabo con una base de datos de 105 piezas dentales y reportaron que no existía diferencia estadísticamente significativa; se concluyó que ambos métodos fueron poco precisos cuando se trató de detectar lesiones de caries a nivel de esmalte; pero a medida que la lesión cariosa fue aumentando en profundidad hacia dentina, la tasa de acierto fue incrementando (14). La presente investigación, encontramos un bajo nivel de concordancia en los diagnósticos de lesiones de caries dental a nivel superficial de esmalte (R1), entre el método convencional y digital.

Hintze y col., compararon tres sistemas digitales de almacenamiento de placa de fósforo: 1) DenOptix (Gendex, Dentsply, Milan, Italia); 2) Cd-dent (Orex, Yokneam, Israel); 3) Digora (Soredex, Hlsinki, Finlandia) y una radiografía convencional Ektaspeed Plus (Eastman Kodak, Rochester, NY, EEUU) expuestos con dos tiempos de exposición diferentes (15). Se comparó su capacidad para detectar lesiones de caries dental proximal en 190 dientes permanentes; se concluyó que el aumento del tiempo de exposición dio lugar a una mayor exactitud en la detección de caries dental, además, reportó diferencias estadísticamente significativas entre los sistemas digitales y el convencional (15). La presente investigación utilizó una base de datos obtenida de radiografías digitales CCD y radiografías convencionales adquiridas con un tiempo de exposición ideal, por lo que sus resultados son similares.

Hintze y Wenzel realizaron un estudio donde evaluaron la precisión del método de diagnóstico para caries dental proximal entre ocho sistemas radiológicos. 1) MPDx (Dental/Medical Diagnostic System Inc., Woodland Hills, CA, EEUU); 2) Dixi (Planmeca, Helsinki, Finlandia); 3) Sidexis (Sirona, Bensheim, 
Alemania); 4) $\mathrm{RVG}_{1}$ (Trophy, Paris, Francia, modelo 1994); 5) RVG (Trophy, Paris, Francia, modelo 2000); 6) Visualix (Gendex, Milan, Italia). Estos seis de tipo digital y dos sistemas de películas convencionales: Ektaspeed Plus e Insight (Eastman Kodak, Rochester, NY, EEUU) para la detección de caries dental en 177 dientes permanentes. Esta investigación presentó diferencias estadísticamente significativas entre los sistemas digitales y convencionales $(\mathrm{p}<0,001)$ (16). Los resultados de este estudio van acorde con los encontrados en la presente investigación.

En conclusión, existió diferencia en el diagnóstico radiológico de lesiones de caries dental proximal usando la técnica de aleta de mordida con radiografía convencional y técnica digital con sistema CCD. Así mismo se encontró una mayor prevalencia de superficies proximales sanas (R0) usando la técnica de aleta de mordida con radiografía convencional y técnica digital CCD, según pieza dentaria y superficie, finalmente hubo mayor concordancia en el diagnóstico radiológico de lesiones de caries dental proximal en la mitad interna de la dentina (R4) y una menor concordancia en la mitad externa del esmalte (R1) usando la técnica de aleta de mordida con radiografias convencionales y técnica digital $\mathrm{CCD}$, según pieza dentaria y superficie. Sin embargo, existieron dos limitaciones fundamentales al realizar el estudio: utilizar una base de datos y no poder afirmar la superioridad de una de las técnicas sobre la otra, por falta de gold estándar histológico, debido a que el presente estudio es parte de una línea de investigación aún en desarrollo, la cual tiene como objetivo la comparación de diversas técnicas diagnósticas para la detección de caries dental, por ello, este primer acercamiento es relevante para indicar que en la práctica clínica existe diferencias en el empleo de ambas técnicas, las cuales deben ser tomadas en cuenta por el profesional.

\section{Correspondencia:}

Francis Marcial López Puerta

Correo electrónico: francis.lopez@upch.pe

\section{REFERENCIAS BIBLIOGRÁFICAS}

1. Russel M, Pitts B. Radiovisiographic diagnosis of dental caries: Initial comparison of basic mode videoprints with bitewing radiographic. Caries Res. 1993;27:65-70.

2. Tyndall DA, Rathore S. Cone Beam- CT Diagnostic Applications: Caries, Periodontal Bone Assessment and endodontic applica- tions. Dent Clin N Am. 2008; 12(52):825-41.

3. Organización Mundial de la Salud. Informe sobre el problema mundial de las enfermedades bucodentales. Ginebra: OMS; 2004

4. Ludlow J, Davies L, White S. Patient risk related to common dental radiographic examinations. J Am Dent Assoc. 2008; 139(9):1237-43.

5. Whaites E. Essentials of dental radiography and radiology. 3ra ed. London: Elsevier Science; 2003.

6. Wenzel A. Digital radiography and caries diagnosis. Dentomaxillofac Radiol. 1998;27: 3-11.

7. Mohtavipour ST, Shahsavari F, Haghighat ASJ, Mohtavipour SS, Malekshoar M. In vitro comparison of conventional film and direct digital radiography in proximal caries detection. J Dentomaxillofac Radiol. 2012;1(2): 1-5.

8. Van Daatselaar A, Van der Stelt F, Weenen J. Effect of number of projections on image quality of local CT. Dentomaxillofac Radiol. 2004; 33(6): 361-9

9. Verdonschot E, Wenzel A, Bronkhorst E. Assessment of diagnostic accuracy in caries detection: an analysis of two methods.Community Dent Oral Epidemiol. 1993; 21(4):203-8.

10. Urzúa R. Técnicas radiograficas dentales y maxilofaciales: Aplicaciones. Venezuela: Amolca; 2005.

11. Khan E, Tyndall D, Caplan D. Extraoral imaging for proximal caries detection: Bitewings vs scanogram. Oral Surg Oral Med Oral Pathol Oral Radiol Endod. 2004; 98(6):730-7.

12. Barbieri G, Flores J, Escribano M, Discepoli N. Actualización en radiología dental. Radiología convencional vs digital. Avances en Odontoestomatología. 2006;22(2): 131-9.

13. Muhammed A, Mohamed I. Subjective image quality comparison between two digital dental radiographic systems and conventional dental film. Saudi Dent J. 2014; 26:145-150.

14. Anupama N, Shashikanth M, Shailesh L. Radiographic assessment of proximal caries: a comparison between film-based and dexis digital imaging systems. J Indian Acad Oral Med Radiol. 2011; 23(3):304-7.

15. Hintze $\mathrm{H}$, Wenzel A, Frydenberg M. Accuracy of caries detection with four storage phosphor systems and E-speed radiographs. Dentomaxillofac Radio.2002;31(3):170-5.

16. Hintze $\mathrm{H}$, Wenzel $\mathrm{A}$. Influence of the validation method on diagnostic accuracy for caries. A comparison of six digital and two conventional radiographic systems. Dentomaxillofac Radio. 2002; 31:44-9.

Recibido : 18/03/2016

Aceptado: 12/05/2016 